\title{
Convection cells induced by spontaneous symmetry breaking
}

\author{
M. Pleimling, B. Schmittmann and R.K.P. Zia \\ 1 Department of Physics, Virginia Tech, Blacksburg, VA 24061-0435 USA
}

PACS 05.70.Ln - Nonequilibrium and irreversible thermodynamics

PACS 47.55.pb - Thermal convection

PACS 64.60.De - Statistical mechanics of model systems

\begin{abstract}
Ubiquitous in nature, convection cells are a clear signature of systems out-ofequilibrium. Typically, they are driven by external forces, like gravity (in combination with temperature gradients) or shear. In this article, we show the existence of such cells in possibly the simplest system, one that involves only a temperature gradient. In particular, we consider an Ising lattice gas on a square lattice, in contact with two thermal reservoirs, one at infinite temperature and another at $T$. When this system settles into a non-equilibrium stationary state, many interesting phenomena exist. One of these is the emergence of convection cells, driven by spontaneous symmetry breaking when $T$ is set below the critical temperature.
\end{abstract}

Introduction. - In nature, convection cells can be found everywhere, existing at length scales from the mi' croscopic to the global. Rayleigh-Benard cells [1] are likely the most well known examples. Another are the vortices (in, e.g., cloud formations) created by the KelvinHelmholtz instability [2, 3. In all cases, external forces drive convection - temperature gradients and gravity in the former case and shear in the latter. Clearly, none of these cells can persist under conditions of thermal equilibrium. Instead, they are distinct signatures of systems far from equilibrium. Motivated by our interest in understanding fundamental issues in non-equilibrium statistical mechanics, we ask here: What are the minimal conditions for convection cells to persist? The answer may provide valuable insight into the essential ingredients associated with physics far from equilibrium. To identify these conditions, we construct minimal systems, seeking the equivalent of the Lenz-Ising model [4 in the study of phase transitions. In this spirit, we focus specifically on non-equilibrium steady states (NESS), in which convection cells persist in a time-independent manner. Here, we present possibly the simplest model with such properties. Unlike the examples above, our system is taken out of equilibrium by a temperature gradient alone. In particular, we couple two (spatial) sectors of an Ising lattice gas to two different thermal baths, evolving with familiar dynamics and rates. Thus, the underlying dynamics fully respect the Ising symmetry. With no other external forces, our cells are the consequence of spontaneous symmetry breaking, i.e., phase segregation.
The properties of the Lenz-Ising model, in its lattice gas version 5, 6, and in thermal equilibrium, are well established. Especially prominent is a second order phase transition at the Onsager [7,8 temperature, $T_{O} \cong 0.5673 \mathrm{~J} / k_{B}$, in half-filled systems in two dimensions (2D). To drive this model into a NESS and explore its behavior far from equilibrium, many authors have introduced a variety of changes to its usual dynamics, e.g., allowing a uniform bias along one of the lattice directions [9, 10] or using two different $T$ 's for exchanges along the two axes [11-14]. A wide range of novel phenomena emerged, leading to a better understanding of NESS in general [15,16]. Our approach here follows similar lines, in that we couple the system to two thermal baths, but in a manner that is far more common in nature. As in earlier studies of NESS, the results are novel and surprising, with the most notable being the emergence of convection cells. In the remainder of this letter, we define our model, present simulation results and quantitative measures for these cells. Next, we provide an exactly solvable (small) system which displays such cells, as well as steps toward a field theoretic framework for analyzing the phenomenon. Deferring systematic details to a future publication, we close with a discussion and an outlook for future studies.

Model specifications. - Our system consists of a 2D Ising model with the usual nearest-neighbor $(\mathrm{NN})$ interaction on a $L_{x} \times L_{y}$ square lattice (with $L_{x}$ and $L_{y}$ both even, for simplicity). In a lattice gas, a site $(x, y)$ may be occupied by a particle or left empty, i.e., $n(x, y)=1$ 
or 0 . The interparticle interactions are attractive, i.e., $\mathcal{H}=-J \sum n(x, y) n\left(x^{\prime}, y^{\prime}\right)$ with the sum over NN sites and $J>0$. The system evolves with ordinary Kawasaki dynamics [17]: A NN pair (also referred to as a "bond") is chosen at random and exchanged according to the familiar Metropolis rates: $\min \left[1, e^{-\Delta \mathcal{H} / k_{B} T}\right]$, where $\Delta \mathcal{H}$ is the change in $\mathcal{H}$ due to the exchange. Specifying the boundary conditions and starting with an initial configuration, this system will settle into thermal equilibrium, with well-known Ising lattice gas properties. In particular, for $T<T_{O}$, a half-filled system displays co-existence of phases, i.e., a high-density domain separated from a low-density one by well defined interfaces. With periodic boundary conditions (PBC), each domain is a strip, generally aligned along an axis so that its interfaces span the smaller of $L_{x}, L_{y}$.

To impose coupling to two thermal baths, we partition the lattice into two sectors (say, the "left" and "right" half-planes: $x \leq L_{x} / 2$ and $>L_{x} / 2$ ) and use different $T$ 's for each 1 This form of drive, with a macroscopic temperature gradient, is obviously ubiquitous, from stove-top cooking to large scale weather patterns. Specifically, we set the right bath temperature, $T^{\prime}$, to infinity (for simplicity), but leave the left bath at a controllable $T$. In other words, if a particle-hole pair lying strictly in the left sector is chosen, they are updated precisely as in the ordinary Ising case. Otherwise, the pair is always exchanged $\left(e^{-\Delta \mathcal{H} / \infty}=1\right)$. A pair straddling the sectors, $\left(L_{x} / 2, y\right)$ $\left(1+L_{x} / 2, y\right)$, is also exchanged (again, for simplicity). As for the boundary conditions, the simplest would be periodic (PBC). However, to facilitate the measurement of convection cells, we impose pinned conditions at the $x$ boundaries. Specifically, we introduce an extra column of immobile objects at $x=0$ : particles at the "bottom" $\left(y \in\left[1, L_{y} / 2\right]\right)$ and holes at the "top" $\left(y \in\left[1+L_{y} / 2, L_{y}\right]\right)$. The $y$-boundaries are periodic. Below, we will discuss how to detect these cells in a system with full PBC. Here, let us emphasize that the pin introduced here plays an identical role for an Ising model in equilibrium, in which the average magentisation of a finite system with full $\mathrm{PBC}$ is zero for all $T$.

In our simulations, a Monte Carlo Step (MCS) consists of $L_{x} L_{y}$ attempts to exchange pairs. Starting with a random, half-filled configuration, we discard $1.6 \times 10^{6}$ MCS so that the system has relaxed into a steady state. The next $K$ MCS are designated as our "run". Mostly, we have $K=3 \times 10^{7}$ and carry out 80 runs for better statistics. In addition to the usual measurements of the average density $\langle n(x, y)\rangle$, we record every successful exchange over the entire run. The latter allows us to define net currents across each bond, e.g., $j_{x}(x, y)$ is the total number of traverses by a particle from $(x, y)$ to $(x+1, y)$, minus such traverses by a hole, divided by $K$. As a check, for each site we compute $\operatorname{div} \vec{j} \equiv j_{x}(x, y)-j_{x}(x-1, y)+j_{y}(x, y)-j_{y}(x, y-1)$ and

\footnotetext{
${ }^{1}$ Other non-equilibrium Ising models coupled to two temperature baths, especially using Glauber spin flip dynamics, have also been studied. For a review and references, see e.g., Section VII-B in [15].
}

see that it is precisely $[n(x, y ; K)-n(x, y ; 0)] / K$. Thus, it vanishes in the $K \rightarrow \infty$ limit.

By contrast, there is no such constraint on the vorticity $\omega \equiv$ curl $\vec{j}$ (a scalar in 2D). On the lattice, $\omega(x, y)$ can be associated with the plaquette centered at $\left(x+\frac{1}{2}, y+\frac{1}{2}\right)$. A discrete version of $\oint \vec{j} \cdot d \vec{\ell}$ around the square,

$\omega(x, y) \equiv j_{x}(x, y)+j_{y}(x+1, y)-j_{x}(x, y+1)-j_{y}(x, y)$

is positive for counter-clockwise circulation. For an equilibrium system, the average current, $\langle\vec{j}\rangle$, must vanish, and so, $\langle\omega\rangle=0$. Being stochastic, $\omega(x, y ; t)$ should be a random walk in time. Thus, with our definition of $\vec{j},\left\langle\omega^{2}\right\rangle$ is expected to vanish as $1 / K$. For a system in a NESS, however, probability currents are non-trivial in general [18.19], so that both $\langle\vec{j}\rangle$ and $\langle\omega\rangle$ may also be non-trivial. Of course, in our model, we do not expect the presence of uniform, global currents or vorticity (such as in the driven lattice gas [9, 10]). Instead, the symmetries dictate the presence of convection cells in equal and opposite pairs.

Simulation results. - Focusing on the existence of $\omega$, we defer our systematic study to a future publication and present only one set of results here, namely, $T \cong 0.88 T_{O}$ with $L_{x}=20,50,100$ and $L_{y}=$ $20,50,100,200,400$. Fig. 1a shows a typical configuration in a $100 \times 100$ system and Fig. $1 \mathrm{~b}$ shows a plot of $\langle n(x, y)\rangle$. Not surprisingly, we find phase segregation in the left sector, with a high-density domain "at the bottom" (due to the pinned BC). Also expected is a homogeneous, halffilled state on the right. Across the sectors, there are density gradients which tend to drive particles/holes from the high/low density domain on the left to the right sector. This leads to emerging local excesses in the right sector which, in turn, are also unsustainable, due to the underlying dynamics, so that we expect nontrivial $\vec{j}, \omega$, and persistent convection cells. Such a scenario is sketched in Fig 1c.

To quantify $\vec{j}$ and $\omega$, we first verify that the net number of exchanges across each bond indeed increases linearly with $K$ for each run. Averaging over the 80 runs, we establish a non-vanishing $\langle\vec{j}(x, y)\rangle$. To display a vector field is typically cumbersome. However, in $2 \mathrm{D}$, a convenient quantity is the scalar stream function $\psi(x, y)$, the curl of which is $\vec{j}$. In Fig. 2a, we show $\psi$ for a $50 \times 50$ system, illustrating that the currents are highly nonlocal, i.e., spread out over the entire latticen! For easier interpretation, we note that $j_{x}=\partial_{y} \psi$ and $j_{y}=-\partial_{x} \psi$. Hence, $\vec{j}$ can be visualized from $\psi$ by taking its gradient and rotating the resultant vectors by $90^{\circ}$, so that the peak at $x=y=25$ corresponds to the center of the $\omega>0$ vortex (red online) in Fig 1c. Another perspective is provided

\footnotetext{
${ }^{2}$ In order to appreciate this remarkable observation, let us emphasize that the currents would be identically zero everywhere, had the two temperatures been the same. Our dynamics violates detailed balance at a local level (three columns of exchanges), yet the currents - a prominent signature of a non-equilibrium system - are nonlocal.
} 

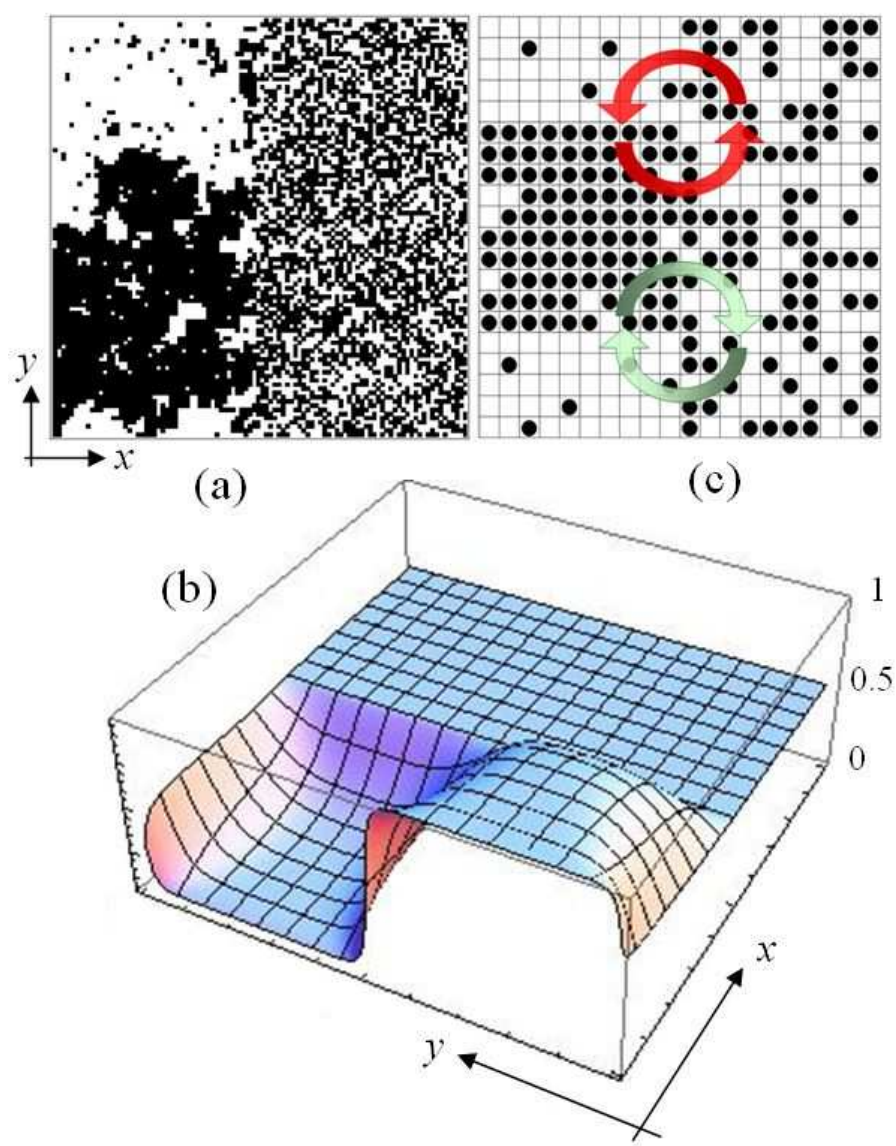

(c)
Fig. 1: (a) Typical configuration of a $100 \times 100$ system, with $T \cong 0.88 T_{O}$ and $T^{\prime}=\infty$. (b) The average density profile. (c) A sketch of a $20 \times 20$ case, illustrating the presence of the convection cells. For clarity, this system is displaced "upwards" by $L_{y} / 4$. Thus, the top row shown here is the $y=15$ line.

by the associated $\omega$, shown in Fig $2 b$. Notably, it is essentially localized at the sector boundary $(x=25)$, with peaks/dips centered on the convection cells. Within each sector, just one or two columns away from the boundary, $\omega$ drops precipitously to noisy backgrounds. To highlight this localization and its secondary characteristics, we illustrate $\omega$ in a $20 \times 400$ system (sector boundary at $x=10$ ). In Fig. 2c, we plot $\omega(x, y)$ against $y$ for several values of $x$. Note that (i) the width of the primary vortex in $y$ is only about 10, (ii) the width in $x$ is $O(1)$, and (iii) there are two secondary, counterflow cells here.

The contrast between $\psi$ and $\omega$ should caution us on how best to describe "non-equilibrium effects" - some aspects being system-wide and others appearing to be localized. Of course, such constrasts are well known: In electrostatics, the potential of a localized charge ranges throughout space. Indeed, the analogy here is precise, since $\nabla^{2} \psi=-\omega$. To emphasize the absence of vortices in an equilibrium system, we consider a model which is superficially similar to ours, by setting $J=0$ on all bonds centered on $x \geq \frac{L_{x}+1}{2}$, but updating with a single $T$ every-
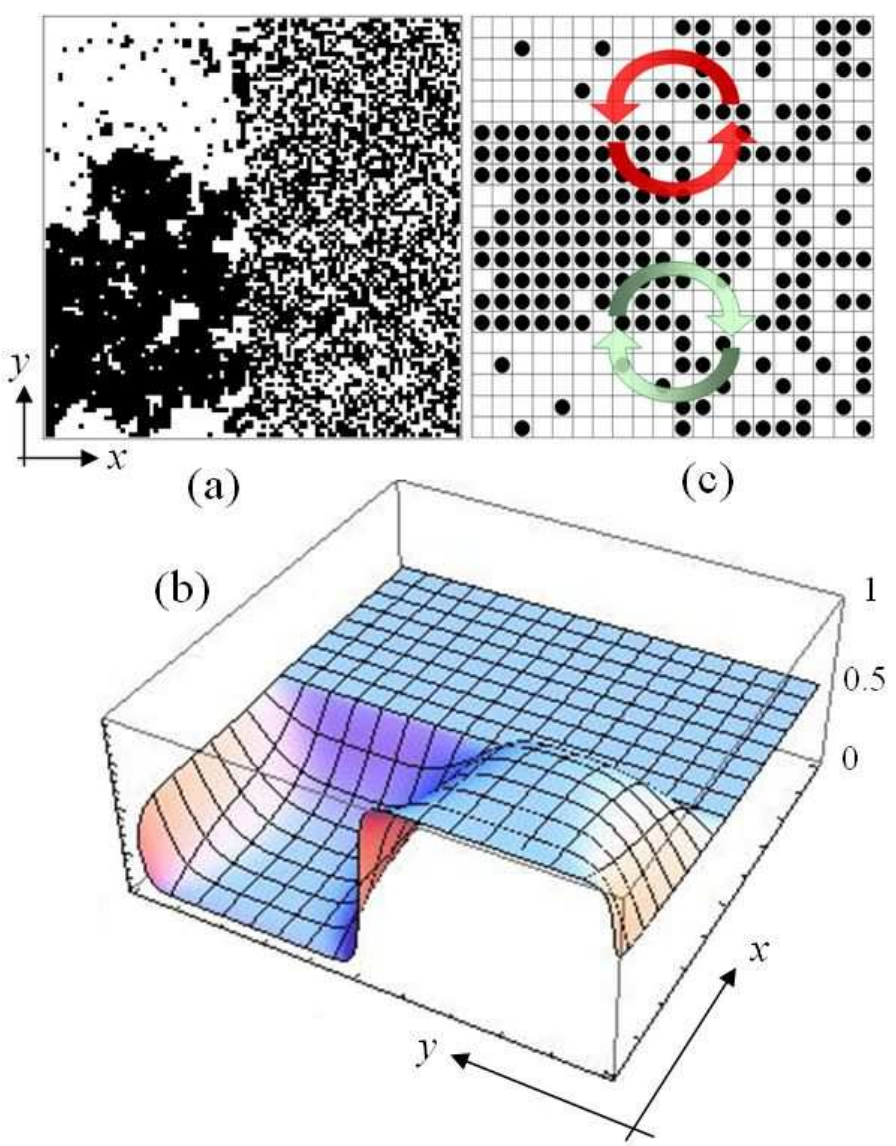

Fig. 2: Persistent currents and vortices present in a $50 \times 50$ system, with $T \cong 0.88 T_{O}$ and $T^{\prime}=\infty$, characterized by (a) the stream function $\psi$ and (b) the vorticity $\omega$. (c) Vorticity in a $20 \times 400$ system: $\omega(x, y)$ vs. $y$ for several values of $x$, showing that it is essentially localized at the sector boundary $(x=10)$, with counter-flow cells about 10 lattice spacings on either side of the primary vortex. Arbitrary units are used for both $\psi$ and $\omega$.

where. The dynamical rules within the right sector here are identical to our model above. The only difference between the two lies with the updates on the three sets of bonds centered on $x=\frac{L_{x}}{2}, \frac{L_{x} \pm 1}{2}$. Simulations (to be reported elsewhere) indeed show all $\omega$ 's distributed around zero, with a width vanishing as $K^{-1 / 2}$.

Theoretical considerations. - To formulate a microscopic theory for our model is facile: A master equation can be written in a line or two. However, to find the stationary distribution is far from trivial. Nevertheless, we can offer some insight by considering the exact solution for the simplest possible case, namely, a $2 \times 2$ lattice with just 2 particles. Now, for just two rows, we impose open $\mathrm{BC}$ in $y$ (since PBC would be meaningless) and so, just one bond is relevant, for coupling and exchange across the rows. With pin $\mathrm{BC}$ in $x$, particle-hole exchanges can take place on only 4 bonds. Thus, their associated $\langle\vec{j}\rangle$ 's in the steady state must share the same magnitude and only a 
single $\omega$ exists. With just 6 configurations $(i=1, \ldots 6)$, it is straightforward to write the master equation and find the stationary probabilities exactly. From here, we can compute the frequency of any exchange to take place and arrive at $\langle\omega\rangle=2\left(1-e^{-4 J / k_{B} T}\right) /\left(7+5 e^{-4 J / k_{B} T}\right)$. To appreciate the significance of having two baths, we repeat this computation with $T^{\prime}<\infty$ and find that the first term in the numerator is $e^{-4 J / k_{B} T^{\prime}}$. Hence, $\langle\omega\rangle$ vanishes when $T^{\prime}=T$, as expected.

A concern may be raised that, together with the open $\mathrm{BC}$, the pinned column affects particles much like gravity and so, our convection cell is no different from those observed daily in the atmosphere. Indeed, for the $2 \times 2$ system, the pin, along with the extremely small size, has serious consequences, such as the vortex persisting for all $T<\infty$. To untangle the effects of pin vs. size, we turn to slightly larger systems, of size $2 \times \ell$ with $\ell \leq 6$, and open $\mathrm{BC}$ at both (left and right) edges. Now, we can re-impose $\mathrm{PBC}$ in $y$ so that translational invariance is restored. Of course, this delocalizes the vortex and the average vorticity, $\langle\omega\rangle$, is necessarily zero. Nevertheless, despite the absence of a gravity-like pin, convection cells are still present and can be detected through a densityvorticity correlation, such as $\langle n \omega\rangle$. Using exact numerical techniques [20, non-trivial values for $\langle n(1, y) \omega(1, y)\rangle$ and $\langle n(1, y) \omega(1, y+1)\rangle$ emerge, persisting again for all finite $T$. We believe that this behavior has a counterpart in an equilibrium Ising model, e.g., in the two-spin correlation $\left\langle s(x, y) s\left(x+\frac{L_{x}}{2}, y+\frac{L_{y}}{2}\right)\right\rangle$. This quantity is non-zero for all $T<\infty$ for a finite system. The difference between the two sectors $T<T_{O}$ and $T \geq T_{O}$ emerges only for $L \rightarrow \infty$ : the correlation vanishes in the latter and remains non-zero for $T<T_{O}$. Of course, a systematic finite-size analysis of $\langle n \omega\rangle$ will address these issues decisively. Simulation studies for this purpose are in progress.

Small systems, though exactly solvable, are clearly of limited value for understanding large scale collective behavior. For the latter, a standard theoretical approach is to exploit Langevin equations for a mesoscopic continuum particle density $\rho(\vec{x}, t)$. For an Ising lattice gas evolving towards thermal equilibrium, a well established route is model B [21]: $\partial_{t} \rho \propto \nabla^{2}(\delta \mathcal{F} / \delta \rho)+\eta$, where $\mathcal{F}$ is the Landau-Ginzburg free energy functional and $\eta$ is a conserved noise. In this approach, the vorticity never appears, since the right hand side is just the divergence of the current. Although curl $\vec{j}$ can never affect the evolution of $\rho$, it can be significant, especially for non-equilibrium systems. However, if we take the form for $\vec{j}$ from model $\mathrm{B}$ above, then (the deterministic part of) curl $\vec{j}$ vanishes identically and cannot be captured. Instead, it is necessary to include the mobility factor, $\sigma$, in the functional form of $\vec{j}$ :

$$
\vec{j}[\rho]=\sigma[\rho]\left\{-\vec{\nabla} \frac{\delta \mathcal{F}}{\delta \rho}\right\} .
$$

Such a factor has been exploited in other studies, especially in systems with external drives like gravitational or electric fields [22,23]. Typically, $\sigma$ is assumed to be $\rho(1-\rho)$ for the lattice gas [15, 16]. To have curl $\vec{j} \neq 0$, a further ingredient is necessary, namely, explicit spatial dependence in either $\sigma$ or $\mathcal{F}$, or both. Otherwise, curl $\vec{j}$ will be proportional to $\vec{\nabla} \rho \times \vec{\nabla} \rho \equiv 0$. In our case, we would model the two sectors coupled to baths with $T \neq T^{\prime}$ by having an explicit $\vec{x}$-dependence in $\mathcal{F}[\rho, \vec{x}]$. (It is also simple to consider models with $\sigma[\rho, \vec{x}]$, which would induce shear when driven.) One advantage of this approach is that we expect $\vec{\nabla} T(x)$ to appear, so that curl $\vec{j}$ will be localized to the sector boundary (for the model presented here). Work is in progress to turn this intuitive picture into a developed mesoscopic theory.

Summary and Outlook. - In this letter, we report the presence of convection cells in a minimal model: an Ising lattice gas coupled to two thermal baths. Unlike cells common in nature, they are not driven by external forces such as gravity or shear. Instead, the microscopic dynamics obeys the full Ising (up-down, or particle-hole) symmetry, so that the convection cells here are induced by the spontaneous breaking of this symmetry (and the ensuing density gradients). Through simulations and an exact analysis of small systems, we investigate a natural quantity to describe convection cells - the vorticity, $\omega$, or curl of the current density. To our knowledge, $\omega$ has never been studied previously in systems where particles are not associated with momenta. We believe our findings offer a new perspective on the vorticity.

A range of interesting questions natural arise from this study, some of which can be explored readily. Are the vortices affected by the details of the exchanges across the sector boundary? How does the spread of $\omega$ along the boundary $\left(O(10)\right.$ in Fig 2c) depend on $L_{x}$ ? Are the properties of the interface (between high- and low-density domains on the left) similar to those in equilibrium, or are they drastically different? Are there any novel critical properties associated with $\omega$, which vanishes along with the spontaneous symmetry breaking at high $T$ ? What is the best characterization of the energy flux from the right to the left sector? Then there are, of course, deeper issues which deserve careful thought and serious effort. What is the origin and behavior of the secondary counterflows? And is there a series of smaller and smaller vortices? How do the phenomena found here behave in the thermodynamic limit? Can the density profiles (e.g., in Fig 1c) be quantitatively understood?

Apart from vorticity and convection cells, our twotemperature model displays many other intriguing and counterintuitive properties. In particular, we found an extremely surprising phenomenon in a fully periodic, $T>T_{O}$ system, in which only a strip of a few columns is coupled to $T^{\prime}=\infty$, namely, the particle density within the strip being a bi-modal distribution (symmetric around $1 / 2$ )! So far, this remarkable behavior is yet to be understood. From such surprises and the presence of convection cells, we can expect this deceptively simple two-temperature lattice gas 
to present us with further puzzling challenges. We believe that solving these puzzles will provide critical clues to formulating an overarching theoretical framework for non-equilibrium statistical mechanics.

We thank Henk Hilhorst and Zoltan Toroczkai for illuminating discussions. This research is supported in part by grants from the US National Science Foundation, DMR-0705152 and DMR-0904999.

\section{REFERENCES}

[1] Chandrasekhar S., Hydrodynamic and Hydromagnetic Stability (Clarendon, Oxford) 1961.

[2] Lord Kelvin, Philos. Mag., 42 (1971) 362.

[3] Helmholtz H., Monatsber. Berlin Akad., 23 (1868) 215.

[4] Ising E., Z. Physik, 31 (1925) 253.

[5] Yang C. N. and Lee T. D., Phys. Rev., 87 (1952) 404.

[6] Lee T. D. and Yang C. N., Phys. Rev., 87 (1952) 410.

[7] Onsager L., Phys. Rev., 65 (1944) 117.

[8] Onsager L., Nuovo Cim. (Suppl.), 6 (1949) 261.

[9] Katz S. and Lebowitz J. L. and Spohn H., Phys. Rev. $B, 28$ (1983) 1655.

[10] Katz S. and Lebowitz J. L. and Spohn H., J. Stat. Phys., 34 (1984) 497.

[11] Garrido L. and Lebowitz J. L. and Maes C. and Spohn H., Phys. Rev. A, 42 (1990) 1954.

[12] Maes C. and Redig F., J. Phys. A, 24 (1991) 4359.

[13] Cheng Z. and Garrido L. and Lebowitz J. L. and VAllés J. L., Europhys. Lett, 14 (1991) 507.

[14] Praestgaard E. and Schmittmann B. and Zia R. K. P., Eur. Phys. J. B, 18 (2000) 675.

[15] Schmittmann B. and ZiA R. K. P., Phase Transitions and Critical Phenomena, edited by C. Domb AND J.L. Lebowitz, Vol. 17 (Academic,London) 1995.

[16] Schmittmann B. and Zia R. K. P., Phys. Rep., 301 (1998) 45.

[17] Kawasaki K., Ann. Phys., 61 (1970) 1.

[18] Zia R. K. P. and Schmittmann B., J. Phys. A, 39 (2006) L407.

[19] Zia R. K. P. and Schmittmann B., J. Stat. Mech.: Theory Exp., (2007) P07012.

[20] Venkat S. and Pleimling M. and Schmittmann B. and ZIA R. K. P., in preparation, ().

[21] Halperin B. I. and Hohenberg P. C. and MA S.-K., Phys. Rev. B, 10 (1974) 139.

[22] Langer J. S. and Baron M. and Miller H. D., Phys. Rev. A, 11 (1975) 1417.

[23] Puri S. and Bray A. J. and Lebowitz J. L., Phys. Rev. E, 56 (1997) 758. 